\title{
EDITORIAL
}

\section{Editorial zum Themenheft Applikationstechnik}

\author{
Jens Karl Wegener ${ }^{1}$
}

๑) Springer-Verlag GmbH Deutschland, ein Teil von Springer Nature 2019

Bei Risiken und Nebenwirkungen fragen Sie bitte Ihren Arzt oder Apotheker.

Wer kennt ihn nicht, diesen Satz. Auch im Bereich des Pflanzenschutzes ist er von Bedeutung. Denn der Einsatz von Pflanzenschutzmitteln und Bioziden ist ebenfalls mit Risiken verbunden, die von den zuständigen Bewertungsbehörden hinsichtlich des Naturhaushalts und der menschlichen Gesundheit bewertet werden.

Aber haben Sie sich schon einmal gefragt, wie diese Risiken und Nebenwirkungen eigentlich bestimmt werden? Grundlage der Bewertung bilden Expositionsdaten, die das Verhalten von Pflanzenschutzmitteln, Beizmitteln oder Bioziden in verschiedenen Anwendungsfällen unter praktischen Umweltbedingungen widerspiegeln. Dafür werden aufwändige technische Versuche zur Bestimmung der Expositionshöhe und deren räumlicher Verteilung durchgeführt. Diese werden u. a. maßgeblich von der eingesetzten Verfahrenstechnik bestimmt. Abdriftmindernde Techniken helfen, die Exposition auf Nicht-Zielflächen deutlich zu reduzieren. Aber auch Technologien, die in der Lage sind, die Anwendung von Pflanzenschutzmitteln zielgerichteter durchzuführen, können die Exposition positiv beeinflussen. Dadurch werden Pflanzenschutzmittel beispielsweise nur dort ausgebracht, wo tatsächlich auch ein Schaderreger aufgetreten ist. Des Weiteren gibt es Systeme, die den Anwender beim Anmischen der Spritzflüssigkeit vor dem direkten Kontakt mit dem unverdünnten Pflanzenschutzmittel schützen. Auch völlig neue Ansätze, wie der „Schneckenroboter", die gänzlich ohne Chemie auskommen, können zukünftig einen wesentlichen Beitrag zur Risikominimierung leisten.

Die in dieser Ausgabe vorgestellten Arbeiten des Instituts für Anwendungstechnik im Pflanzenschutz des Julius Kühn-Instituts zeigen einen Querschnitt technischer Lösungsmöglichkeiten und neuer Bewertungsverfahren, um die Risiken und Nebenwirkungen des Pflanzenschutzes weiter zu reduzieren. Sollte die Lektüre Fragen aufwerfen, wenden Sie sich bitte einfach an den Anwendungstechniker Ihres Vertrauens.

Interessenkonflikt J.K. Wegener gibt an, dass kein Interessenkonflikt besteht.
Jens Karl Wegener

jens-karl.wegener@julius-kuehn.de

1 Institut für Anwendungstechnik im Pflanzenschutz, Julius Kühn-Institut, Messeweg 11/12, 38104 Braunschweig, Deutschland 